\title{
Zinc Finger E-Box-Binding Homeobox 1
}

National Cancer Institute

\section{Source}

National Cancer Institute. Zinc Finger E-Box-Binding Homeobox 1. NCI Thesaurus. Code C105607.

Zinc finger E-box-binding homeobox 1 (1124 aa, 124 kDa) is encoded by the human ZEB1 gene. This protein is involved in both the transcriptional repression of interleukin 2 and the positive regulation of neuronal differentiation. 